\title{
Article \\ New Theorems for Oscillations to Differential Equations with Mixed Delays
}

\author{
Shyam Sundar Santra ${ }^{1, *,+} \mathbb{D}$, Debasish Majumder ${ }^{1,+}$, Rupak Bhattacharjee ${ }^{1,+}$, Omar Bazighifan ${ }^{2, *,+} \mathbb{D}$, \\ Khaled Mohamed Khedher $3,4,+(\mathbb{D})$ and Marin Marin ${ }^{5, *,+} \mathbb{D}$
}

1 Department of Mathematics, JIS College of Engineering, Kalyani 741235, India; debasish.majumder@jiscollege.ac.in (D.M.); rupak.bhattacharjee@jiscollege.ac.in (R.B.)

2 Department of Mathematics, Faculty of Science, Hadhramout University, Hadhramout 50512, Yemen

3 Department of Civil Engineering, College of Engineering, King Khalid University, Abha 61421, Saudi Arabia; kkhedher@kku.edu.sa

4 Department of Civil Engineering, High Institute of Technological Studies, Mrezgua University Campus, Nabeul 8000, Tunisia

5 Department of Mathematics and Computers Science, Transilvania University of Brasov, B-dul Eroilor 29, 500036 Brasov, Romania

* Correspondence: shyamsundar.santra@jiscollege.ac.in (S.S.S.); o.bazighifan@hu.edu.ye (O.B.); m.marin@unitbv.ro (M.M.)

+ These authors contributed equally to this work.

Citation: Santra, S.S.; Majumder, D.; Bhattacharjee, R.; Bazighifan, O.; Khedher, K.M.; Marin, M. New Theorems for Oscillations to Differential Equations with Mixed Delays. Symmetry 2021, 13, 367. https://doi.org/10.3390/sym13030367

Academic Editor: Youssef N. Raffoul

Received: 8 February 2021

Accepted: 23 February 2021

Published: 25 February 2021

Publisher's Note: MDPI stays neutral with regard to jurisdictional claims in published maps and institutional affiliations.

Copyright: (c) 2021 by the authors. Licensee MDPI, Basel, Switzerland. This article is an open access article distributed under the terms and conditions of the Creative Commons Attribution (CC BY) license (https:// creativecommons.org/licenses/by/ $4.0 /)$.

\begin{abstract}
The oscillation of differential equations plays an important role in many applications in physics, biology and engineering. The symmetry helps to deciding the right way to study oscillatory behavior of solutions of this equations. The purpose of this article is to establish new oscillatory properties which describe both the necessary and sufficient conditions for a class of nonlinear second-order differential equations with neutral term and mixed delays of the form $\left(p(\iota)\left(w^{\prime}(\iota)\right)^{\alpha}\right)^{\prime}+r(\iota) u^{\beta}(\nu(\iota))=0, \iota \geq \iota_{0}$ where $w(\iota)=u(\iota)+q(\iota) u(\zeta(\iota))$. Furthermore, examining the validity of the proposed criteria has been demonstrated via particular examples.
\end{abstract}

Keywords: Lebesgue's dominated convergence theorem; neutral; oscillation; nonoscillation; non-linear

\section{Introduction}

In this paper we present our work in the study of certain oscillation properties of second-order differential equations containing mixed delays.

Nowadays, the analysis of qualitative properties of ordinary differential equations is attracting considerable attention from the scientific community due to numerous applications in several contexts as Biology, Physics, Chemistry, and Dynamical Systems. For some details related the recent studies on oscillation and non-oscillation properties, exponential stability, instability, existence of unbounded solutions of the equations under consideration, we refer the reader to the books [1,2]. It is worth pointing out that both oscillation and stability criteria are currently used in the studies of nonlinear mathematical models with delay for single species and several species with interactions, in logistic models, $\alpha$-delay models, mathematical models with varying capacity, mathematical models for food-limited population dynamics with periodic coefficients, diffusive logistic models (for instance, diffusive Malthus-type models with several delays, autonomous diffusive delayed logistic models with Neumann boundary conditions, periodic diffusive logistic Volterra-type models with delays, and so on). In the last few years, the research activity concerning the oscillation of solutions of neutral differential equations has been received considerable attention. Moreover, neutral equations contribute to many applications in economics, physics, medicine, engineering and biology, see [3-8]. The literature is full of very interesting results linked with the oscillation properties for second-order differential equations. Now we recall some studies that have a strong connection with the content of this paper. 
In [9], the authors obtained some oscillation criteria of the following second-order neutral differential equations

$$
\left(p(\iota)[u(\iota)+q(\iota) u(\zeta(\iota))]^{\prime}\right)^{\prime}+r(\iota) u(\sigma(\iota))+v(\iota) u(\eta(\iota))=0
$$

considering the cases in which the arguments are delayed, advanced or mixed. In [10], the authors had investigated some oscillation properties of the solutions of the following equation

$$
\left(p(\iota) z^{\prime}(\iota)\right)^{\prime}+r(\iota) u(\sigma(\iota))=0, \quad \iota \geq \iota_{0} \geq 0,
$$

where $z(\iota)=u(\iota)+a(\iota) u(\iota-\tau)+b(\iota) u(\iota+\delta)$. It is interesting to notice that, in the aforementioned works, the authors obtained only sufficient conditions that ensure the oscillation of the solutions of the considered equations. A problem worthy of investigations is the study of necessary and sufficient conditions for oscillation, and some satisfactory answers were given in [11-18]. Finally, the interested readers are referred to the following papers and to the references therein for some recent results on the oscillation theory for ordinary differential equations of several orders [19-27].

In this work, we obtained the necessary and sufficient conditions for the oscillation of solutions to second-order non-linear differential equations in the form

$$
\left(p(\iota)\left(w^{\prime}(\iota)\right)^{\alpha}\right)^{\prime}+r(\iota) u^{\beta}(v(\iota))=0, \quad \iota \geq \iota_{0},
$$

where

$$
w(\iota)=u(\iota)+q(\iota) u(\zeta(\iota)) .
$$

The functions $r, p, q, v, \zeta$ are continuous and satisfy the conditions stated below;

(a) $\quad v \in C([0, \infty), \mathbb{R}), \zeta \in C^{2}([0, \infty), \mathbb{R}), v(\iota)<\iota, \zeta(\iota)<\iota, \lim _{\iota \rightarrow \infty} v(\iota)=\infty$, $\lim _{l \rightarrow \infty} \zeta(\iota)=\infty$.

(b) $\quad v \in C([0, \infty), \mathbb{R}), \zeta \in C^{2}([0, \infty), \mathbb{R}), v(\iota)>\iota, \zeta(\iota)<\iota, \lim _{l \rightarrow \infty} v(\iota)=\infty$, $\lim _{\iota \rightarrow \infty} \zeta(\iota)=\infty$.

(c) $p \in C^{1}([0, \infty), \mathbb{R}), r, \tilde{r} \in C([0, \infty), \mathbb{R}) ; 0<p(\iota), 0 \leq r(\iota), 0 \leq \tilde{r}(\iota)$, for all $\iota \geq 0$.

(d) $q \in C^{2}\left([0, \infty), \mathbb{R}_{+}\right)$with $0 \leq q(\iota) \leq a<1$.

(e) $\lim _{\iota \rightarrow \infty} P(\iota)=\infty$ where $P(\iota)=\int_{0}^{\iota} p^{-1 / \alpha}(s) \mathrm{d} s$.

(f) $\alpha$ and $\beta$ are the quotient of two positive odd integers.

\section{Preliminary Results}

To make our notations simpler, we set

$$
R_{1}(\iota)=r(\iota)((1-a) w(v(\iota)))^{\beta} .
$$

Lemma 1. Suppose (a)-(f) holds for $\iota \geq \iota_{0}$, and if $u$ is an eventually positive solution of (1). Then $w$ satisfies

$$
0<w(\iota), \quad w^{\prime}(\iota)>0, \quad \text { and } \quad\left(p(\iota)\left(w^{\prime}(\iota)\right)^{\alpha}\right)^{\prime} \leq 0 \quad \text { for } \quad \iota \geq \iota_{1} .
$$

Proof. Let $u$ be an eventually positive solution. Then $w(\iota)>0$ and there exists $\iota_{0} \geq 0$ such that $u(\iota)>0, u(v(\iota))>0, u(\zeta(\iota))>0$ for all $\iota \geq \iota_{0}$. Then (1) gives that

$$
\left(p(\iota)\left(w^{\prime}(\iota)\right)^{\alpha}\right)^{\prime}=-r(\iota) u^{\beta}(v(\iota)) \leq 0
$$

which shows that $p(\iota)\left(w^{\prime}(\iota)\right)^{\alpha}$ is non-increasing for $\iota \geq \iota_{0}$. Next we claim that for $w>0$, $p(\iota)\left(w^{\prime}(\iota)\right)^{\alpha}$ is positive for $\iota \geq \iota_{1}>\iota_{0}$. If not, let $p(\iota)\left(w^{\prime}(\iota)\right)^{\alpha} \leq 0$ for $\iota \geq \iota_{1}$, we can choose $c>0$ such that

$$
p(\iota)\left(w^{\prime}(\iota)\right)^{\alpha} \leq-c,
$$

that is,

$$
w^{\prime}(\iota) \leq(-c)^{1 / \alpha} p^{-1 / \alpha}(\iota)
$$


Integrating both sides from $\iota_{1}$ to $\iota$ we get

$$
w(\iota)-w\left(\iota_{1}\right) \leq(-c)^{1 / \alpha}\left(P(\iota)-P\left(\iota_{1}\right)\right) .
$$

Taking limit both sides as $\iota \rightarrow \infty$, we have $\lim _{\iota \rightarrow \infty} w(\iota) \leq-\infty$ which leads to a contradiction to $w(\iota)>0$. Hence, $\quad p(\iota)\left(w^{\prime}(\iota)\right)^{\alpha}>0$ for $\iota \geq \iota_{1}$ i.e., $w^{\prime}(\iota)>0$ for $\iota \geq \iota_{1}$. Hence proved.

Lemma 2. Suppose (a)-(f) hold for $\iota \geq \iota_{0}$, and if $u$ is an eventually positive solution of (1). Then $w$ satisfies

$$
u(\iota) \geq(1-a) w(\iota) \text { for } \iota \geq \iota_{1} .
$$

Proof. Assume that $u$ be an eventually positive solution of (1). Then $w(\iota)>0$ and there exists $\iota \geq \iota_{1}>\iota_{0}$ such that

$$
\begin{aligned}
u(\iota) & =w(\iota)-q(\iota) u(\zeta(\iota)) \\
& \geq w(\iota)-q(\iota) w(\zeta(\iota)) \\
& \geq w(\iota)-q(\iota) w(\iota) \\
& =(1-q(\iota)) w(\iota) \\
& \geq(1-a) w(\iota) .
\end{aligned}
$$

Hence $w$ satisfies (4) for $\iota \geq \iota_{1}$.

Remark 1. The above two lemmas hold for any $\alpha$ and $\beta$ (i.e., $\alpha \geq \beta$ or $\alpha \leq \beta$ ).

\section{Main Results}

Theorem 1. Let (b)-(f) hold for $\iota \geq \iota_{0}$ and $\beta>\alpha$. Then every solution of (1) is oscillatory if and only if

$$
\int_{0}^{\infty} p^{-1 / \alpha}(s)\left[\int_{s}^{\infty} r(\psi) \mathrm{d} \psi\right]^{1 / \alpha} \mathrm{d} s=\infty
$$

Proof. Let $u$ is an eventually positive solution of (1). Then $w(\iota)>0$ and there exists $\iota_{0} \geq 0$ such that $u(\iota)>0, u(v(\iota))>0, u(\zeta(\iota))>0$ for all $\iota \geq \iota_{0}$. Thus, Lemmas 1 and 2 holds for $\iota \geq \iota_{1}$. By Lemma 1, there exists $\iota_{2}>\iota_{1}$ such that $w^{\prime}(\iota)>0$ for all $\iota \geq \iota_{2}$. Then there exists $\iota_{3}>\iota_{2}$ and $c>0$ such that $w(\iota) \geq c$ for all $\iota \geq \iota_{3}$. Next using Lemma 2, we wet $u(\iota) \geq(1-a) w(\iota)$ for all $\iota \geq \iota_{3}$ and (1) become

$$
\left(p(\iota)\left(w^{\prime}(\iota)\right)^{\alpha}\right)^{\prime}+R_{1}(\iota) \leq 0
$$

Integrating (6) from $\iota$ to $\infty$ we get

$$
\left[p(s)\left(w^{\prime}(s)\right)^{\alpha}\right]_{\iota}^{\infty}+\int_{l}^{\infty} R_{1}(s) \mathrm{d} s \leq 0
$$

Since $p(\iota)\left(w^{\prime}(\iota)\right)^{\alpha}$ is positive and non-decreasing, $\lim _{\iota \rightarrow \infty} p(\iota)\left(w^{\prime}(\iota)\right)^{\alpha}$ finitely exists and is positive.

$$
p(\iota)\left(w^{\prime}(\iota)\right)^{\alpha} \geq \int_{\iota}^{\infty} R_{1}(s) \mathrm{d} s,
$$

that is,

$$
\begin{aligned}
w^{\prime}(\iota) & \geq p^{-1 / \alpha}(\iota)\left[\int_{\iota}^{\infty} R_{1}(s) \mathrm{d} s\right]^{1 / \alpha} \\
& =(1-a)^{\beta / \alpha} p^{-1 / \alpha}(\iota)\left[\int_{\iota}^{\infty} r(s) w^{\beta}(v(s)) \mathrm{d} s\right]^{1 / \alpha} .
\end{aligned}
$$


Using the assumption (b) and $w(\iota)$ is non-decreasing,

$$
w^{\prime}(\iota) \geq(1-a)^{\beta / \alpha} p^{-1 / \alpha}(\iota)\left[\int_{\iota}^{\infty} r(s) \mathrm{d} s\right]^{1 / \alpha} w^{\beta / \alpha}(\iota),
$$

that is,

$$
\frac{w^{\prime}(\iota)}{w^{\beta / \alpha}(\iota)} \geq(1-a)^{\beta / \alpha} p^{-1 / \alpha}(\iota)\left[\int_{\iota}^{\infty} r(s) \mathrm{d} s\right]^{1 / \alpha} .
$$

Taking integration both sides from $\iota_{3}$ to $\infty$ we have,

$$
(1-a)^{\beta / \alpha} \int_{l_{3}}^{\infty} p^{-1 / \alpha}(s)\left[\int_{s}^{\infty} r(\psi) \mathrm{d} \psi\right]^{1 / \alpha} \mathrm{d} s \leq \int_{\iota_{3}}^{\infty} \frac{w^{\prime}(s)}{w^{\beta / \alpha}(s)} \mathrm{d} s<\infty
$$

due to $\beta>\alpha$, which is a contradiction to (5) and hence the sufficient part of the theorem is proved.

Next by applying contrapositive argument we proved the necessary part. If (5) does not hold, then for every $\varepsilon>0$ there exists $\iota \geq \iota_{0}$ for which

$$
\int_{l}^{\infty} p^{-1 / \alpha}(s)\left[\int_{s}^{\infty} r(\psi) \mathrm{d} \psi\right]^{1 / \alpha} \mathrm{d} s<\varepsilon \quad \text { for } \quad \iota \geq T,
$$

where $2 \varepsilon=\left[\frac{1}{1-a}\right]^{-\beta / \alpha}>0$. Let us define a set

$$
V=\left\{u \in C([0, \infty)): \frac{1}{2} \leq u(\iota) \leq \frac{1}{1-a} \text { for all } \iota \geq T\right\}
$$

and $\Phi: V \rightarrow V$ as

$$
(\Phi u)(\iota)= \begin{cases}0 & \text { if } \iota \leq T, \\ \frac{1+a}{2(1-a)}-q(\iota) u(\zeta(\iota)) & \\ +\int_{\iota}^{\iota} p^{-1 / \alpha}(s)\left[\int_{s}^{\infty} r(\psi) u^{\beta}(v(\psi)) \mathrm{d} \psi\right]^{1 / \alpha} \mathrm{d} s & \text { if } \iota>T .\end{cases}
$$

Next we prove $(\Phi u)(\iota) \in V$. For $u(\iota) \in V$,

$$
\begin{aligned}
(\Phi u)(\iota) & \leq \frac{1+a}{2(1-a)}+\int_{T}^{\iota} p^{-1 / \alpha}(s)\left[\int_{s}^{\infty} r(\psi)\left(\frac{1}{1-a}\right)^{\beta} \mathrm{d} \psi\right]^{1 / \alpha} \mathrm{d} s \\
& \leq \frac{1+a}{2(1-a)}+\left(\frac{1}{1-a}\right)^{\beta / \alpha} \times \varepsilon \\
& =\frac{1+a}{2(1-a)}+\frac{1}{2}=\frac{1}{1-a}
\end{aligned}
$$

and further, for $u(\iota) \in V$

$$
(\Phi u)(\iota) \geq \frac{1+a}{2(1-a)}-q(\iota) \times \frac{1}{1-a}+0 \geq \frac{1+a}{2(1-a)}-\frac{a}{1-a}=\frac{1}{2} .
$$

Hence, $\Phi$ maps from $V$ to $V$.

Now we are going to find a fixed point for $\Phi$ in $V$ which will eventually give a positive solution of (1). 
First, we define a sequence of functions in $\mathrm{V}$ by

$$
\begin{gathered}
u_{0}(\iota)=0 \quad \text { for } \iota \geq \iota_{0}, \\
u_{1}(\iota)=\left(\Phi u_{0}\right)(\iota)= \begin{cases}0 & \text { if } \iota<T \\
\frac{1}{2} & \text { if } \iota \geq T^{\prime}\end{cases} \\
u_{n+1}(\iota)=\left(\Phi u_{n}\right)(\iota) \text { for } n \geq 1, \iota \geq T .
\end{gathered}
$$

Here, we see $u_{1}(\iota) \geq u_{0}(\iota)$ for each fixed $\iota$ and $\frac{1}{2} \leq u_{n-1}(\iota) \leq u_{n}(\iota) \leq \frac{1}{1-a}, \quad \iota \geq T$ for all $n \geq 1$. Thus, $u_{n}$ converges point-wise to a function $u$. By Lebesgue's Dominated Convergence Theorem $u$ is a fixed point of $\Phi$ in $V$, which shows that there has a nonoscillatory solution. This completes the proof of the theorem.

Theorem 2. Let (a), (c)-(f) hold for $\iota \geq \iota_{0}$ and $\beta<\alpha$. Then every solution of (1) is oscillatory if and only if

$$
\int_{0}^{\infty} r(\psi)[(1-a) P(\nu(\psi))]^{\beta} \mathrm{d} \psi=\infty
$$

Proof. Let $u(\iota)$ be an eventually positive solution of (1). Then proceeding as in Theorem 1 , we have $\iota_{2}>\iota_{1}>\iota_{0}$ such that (7) holds for all $\iota \geq \iota_{2}$. Using (e), there exists $\iota_{3}>\iota_{2}$ for which $P(\iota)-P\left(\iota_{3}\right) \geq \frac{1}{2} P(\iota)$ for $\iota \geq \iota_{3}$. Integrating (7) from $\iota_{3}$ to $\iota$, we have

$$
\begin{aligned}
w(\iota)-w\left(\iota_{3}\right) & \geq \int_{\iota_{3}}^{\iota} p^{-1 / \alpha}(s)\left[\int_{s}^{\infty} R_{1}(\kappa) \mathrm{d} \kappa\right]^{1 / \alpha} \mathrm{d} s \\
& \geq \int_{\iota_{3}}^{\iota} p^{-1 / \alpha}(s)\left[\int_{\iota}^{\infty} R_{1}(\kappa) \mathrm{d} \kappa\right]^{1 / \alpha} \mathrm{d} s,
\end{aligned}
$$

that is,

$$
\begin{aligned}
w(\iota) & \geq\left(P(\iota)-P\left(\iota_{3}\right)\right)\left[\int_{\iota}^{\infty} R_{1}(\kappa) \mathrm{d} \kappa\right]^{1 / \alpha} \\
& \geq \frac{1}{2} P(\iota)\left[\int_{\iota}^{\infty} R_{1}(\kappa) \mathrm{d} \kappa\right]^{1 / \alpha} .
\end{aligned}
$$

Hence,

$$
w(\iota) \geq \frac{1}{2} P(\iota) U^{1 / \alpha}(\iota) \quad \text { for } \iota \geq \iota_{3}
$$

where

$$
U(\iota)=\int_{\iota}^{\infty} r(\kappa)((1-a) w(v(\kappa)))^{\beta} \mathrm{d} \kappa
$$

Now,

$$
\begin{aligned}
U^{\prime}(\iota) & =-r(\iota)((1-a) w(v(\iota)))^{\beta} \\
& \leq-\frac{1}{2^{\beta}} r(\iota)[(1-a) P(\nu(\iota))]^{\beta} U^{\beta / \alpha}(v(\iota)) \leq 0
\end{aligned}
$$

which shows that $U(\iota)$ is non-increasing on $\left[\iota_{4}, \infty\right)$ and $\lim _{\iota \rightarrow \infty} U(\iota)$ exists. Using (10) and (a), we find 


$$
\begin{aligned}
{\left[U^{1-\beta / \alpha}(\iota)\right]^{\prime} } & =(1-\beta / \alpha) U^{-\beta / \alpha}(\iota) U^{\prime}(\iota) \\
& \leq-\frac{1-\beta / \alpha}{2^{\beta}} r(\iota)[(1-a) P(v(\iota))]^{\beta} U^{\beta / \alpha}(v(\iota)) U^{-\beta / \alpha}(\iota) \\
& \leq-\frac{1-\beta / \alpha}{2^{\beta}} r(\iota)[(1-a) P(v(\iota))]^{\beta} .
\end{aligned}
$$

Integrating (11) from $\iota_{3}$ to $\iota$ we have,

$$
\left[U^{1-\beta / \alpha}(s)\right]_{\iota_{4}}^{\iota} \leq-\frac{1-\beta / \alpha}{2^{\beta}} \int_{\iota_{3}}^{\iota} r(s)[(1-a) P(v(s))]^{\beta} \mathrm{d} s,
$$

that is,

$$
\left.\frac{1-\beta / \alpha}{2^{\beta}}\left[\int_{0}^{\infty} r(s)[(1-a) P(v(s))]^{\beta} \mathrm{d} s\right]^{\beta}\right] \leq-\left[U^{1-\beta / \alpha}(s)\right]_{\iota_{3}}^{\iota}<U^{1-\beta / \alpha}\left(\iota_{3}\right)<\infty
$$

which contradicts (8). This completes the proof of the theorem.

Example 1. Consider the neutral differential equations

$$
\left(\left(\left(u(\iota)+e^{-\iota} u(\zeta(\iota))\right)^{\prime}\right)^{1 / 3}\right)^{\prime}+\iota(u(\iota+2))^{5 / 3}=0 .
$$

Here $\alpha=1 / 3, p(\iota)=1,0<q(\iota)=e^{-\iota}<1, v(\iota)=\iota+2$. For $\beta=5 / 3$, we have $\beta=5 / 3>\alpha=1 / 3$. To check (5) we have

$$
\int_{\iota_{0}}^{\infty}\left[\frac{1}{p(s)}\left[\int_{s}^{\infty} r(\psi) d \psi\right]\right]^{1 / \alpha} d s=\int_{2}^{\infty}\left[\int_{s}^{\infty} \psi d \psi\right]^{3} d s=\infty .
$$

So, all the conditions of of Theorem 1 hold. Thus, each solution of (12) is oscillatory.

Example 2. Consider the neutral differential equations

$$
\left(e^{-\iota}\left(\left(u(\iota)+e^{-\iota} u(\zeta(\iota))\right)^{\prime}\right)^{11 / 3}\right)^{\prime}+\frac{1}{\iota+1}(u(\iota-2))^{7 / 3}=0 .
$$

Here $\alpha=11 / 3, p(\iota)=e^{-\iota}, 0<q(\iota)=e^{-\iota}<1, v(\iota)=\iota-2, P(\iota)=\int_{0}^{\iota} e^{3 s / 11} d s=$ $\frac{11}{3}\left(e^{3 l / 11}-1\right)$. For $\beta=7 / 3$, we get $\beta=7 / 3<\alpha=11 / 3$. To check (8) we have

$$
\begin{aligned}
& \frac{1}{(2)^{\beta}}\left[\int_{0}^{\infty} r(\psi)[(1-a) P(v(\psi))]^{\beta} d \psi\right] \\
& =\frac{1}{(2)^{7 / 3}} \int_{0}^{\infty} \frac{1}{\psi+1}\left[(1-a) \frac{11}{3}\left(e^{3(\psi-2) / 11}-1\right)\right]^{7 / 3} d \psi=\infty .
\end{aligned}
$$

So, all the conditions of Theorem 2 hold, and therefore, each solution of (13) is oscillatory.

\section{Conclusions}

In this work, we studied second order highly nonlinear neutral differential equations and established necessary and sufficient conditions for the oscillation of (1) when the neutral coefficient lies in $[0,1)$. We already studied this for the case when $-1 \leq q(\iota) \leq 0$. The obtained method is applicable for any type of second-order delay differential equation. In this direction, we have an open problem, namely: "Can we find the necessary and sufficient conditions for the oscillation of the solutions to the equations (1) for the range $-\infty<q(\iota)<-1$ or $1<q(\iota)<\infty$ ?". 
Author Contributions: Conceptualization, S.S.S., D.M., R.B., O.B., K.M.K. and M.M. These authors contributed equally to this work. All authors have read and agreed to the published version of the manuscript.

Funding: This research work was supported by the Deanship of Scientific Research at King Khalid University under grant number RGP. 2/100/42.

Institutional Review Board Statement: Not applicable.

Informed Consent Statement: Not applicable.

Data Availability Statement: Not applicable.

Acknowledgments: The authors thank the reviewers for their useful comments, which led to the improvement of the content of the paper. Khaled Mohamed Khedher would like to thank the Deanship of Scientific Research at King Khalid University for funding this work through the large research groups under grant number RGP.2/100/42.

Conflicts of Interest: The authors declare no conflict of interest.

\section{References}

1. Berezansky, L.; Domoshnitsky, A.; Koplatadze, R. Oscillation, Nonoscillation, Stability and Asymptotic Properties for Second and Higher Order Functional Differential Equations; Chapman \& Hall/CRC Press: Boca Raton, FL, USA, 2020.

2. Agarwal, R.P.; O’Regan, D.; Saker, S.H. Oscillation and Stability of Delay Models in Biology; Springer International Publishing: New York, NY, USA, 2014.

3. Ottesen, J.T. Modelling of the Baroreflex-Feedback Mechanism with Time-Delay. J. Math. Biol. 1997, 36, 41-63. [CrossRef]

4. Bazighifan, O.; Abdeljawad, T.; Al-Mdallal, Q.M. Differential equations of even-order with $p$-Laplacian like operators: Qualitative properties of the solutions. Adv. Differ. Equ. 2021, 2021, 96. [CrossRef]

5. Agarwal, R.P.; Bohner, M.; Li, T.; Zhang, C. Oscillation of second-order differential equations with a sublinear neutral term. Carpathian J. Math. 2014, 30, 1-6.

6. Bhatti, M.M.; Marin, M.; Zeeshan, A.; Ellahi, R.; Abdelsalam, S.I. Swimming of Motile Gyrotactic Microorganisms and Nanoparticles in Blood Flow Through Anisotropically Tapered Arteries. Front. Phys. 2020, 8, 95. [CrossRef]

7. Marin, M.; Vlase, S.; Paun, M. Considerations on double porosity structure for micropolar bodies. Aip Adv. 2015, 5, 037113. [CrossRef]

8. Khan, A.A.; Bukhari, S.R.; Marin, M.; Ellahi, R. Effects of chemical reaction on third-grade MHD fluid flow under the influence of heat and mass transfer with variable reactive index. Heat Transf. Res. 2019, 50, 1061-1080. [CrossRef]

9. Baculíková, B.; Li, T.; Džurina, J. Oscillation theorems for second order neutral differential equations. Electron. J. Qual. Theory Differ. Equations 2011, 63, 1-13. [CrossRef]

10. Arul, R.; Shobha, V.S. Oscillation of second order neutral differential equations with mixed neutral term. Int. J. Pure Appl. Math. 2015, 104, 181-191. [CrossRef]

11. Pinelas, S.; Santra, S.S. Necessary and sufficient condition for oscillation of nonlinear neutral first-order differential equations with several delays. J. Fixed Point Theory Appl. 2018, 20, 27. [CrossRef]

12. Bazighifan, O.; Ruggieri, M.; Santra, S.S.; Scapellato, A. Qualitative Properties of Solutions of Second-Order Neutral Differential Equations. Symmetry 2020, 12, 1520. [CrossRef]

13. Santra, S.S.; Bazighifan, O.; Ahmad, H.; Chu, Y.M. Second-Order Differential Equation: Oscillation Theorems and Applications. Math. Probl. Eng. 2020, 2020, 8820066. [CrossRef]

14. Santra, S.S.; Bazighifan, O.; Ahmad, H.; Yao, S.W. Second-Order Differential Equation with Multiple Delays: Oscillation Theorems and Applications. Complexity 2020, 2020, 8853745. [CrossRef]

15. Tripathy, A.K.; Santra, S.S. Necessary and sufficient conditions for oscillation of second-order differential equations with nonpositive neutral coefficients. Math. Bohem. 2020. [CrossRef]

16. Santra, S.S. Necessary and sufficient conditions for oscillation to second-order half-linear delay differential equations. J. Fixed Point Theory Appl. 2019, 21, 85. [CrossRef]

17. Santra, S.S.; Ghosh, T.; Bazighifan, O. Explicit criteria for the oscillation of second-order differential equations with several sub-linear neutral coefficients. Adv. Differ. Equ. 2020, 2020, 643. [CrossRef]

18. Santra, S.S.; Alotaibi, H.; Bazighifan, O. On the qualitative behavior of the solutions to second-order neutral delay differential equations. J. Inequalities Appl. 2020, 2020, 643. [CrossRef]

19. Santra, S.S.; Nofal, T.A.; Alotaibi, H.; Bazighifan, O. Oscillation of Emden-Fowler-type neutral delay differential equations. Axioms 2020, 9, 136. [CrossRef]

20. Bazighifan, O.; Ruggieri, M.; Scapellato, A. An Improved Criterion for the Oscillation of Fourth-Order Differential Equations. Mathematics 2020, 8, 610. [CrossRef]

21. Karpuz, B.; Santra, S.S. New criteria for the oscillation and asymptotic behavior of second-order neutral differential equations with several delays. Turk. J. Math. 2020, 44, 1990-2003. [CrossRef] 
22. Santra, S.S. Necessary and sufficient conditions for oscillation of second-order delay differential equations. Tatra Mt. Math. Publ. 2020, 75, 135-146. [CrossRef]

23. Agarwal, R.P.; Bazighifan, O.; Ragusa, M.A. Nonlinear Neutral Delay Differential Equations of Fourth-Order: Oscillation of Solutions. Entropy 2021, 23, 129. [CrossRef] [PubMed]

24. Li, T.; Rogovchenko, Y.V. Oscillation theorems for second-order nonlinear neutral delay differential eqquations. Abstr. Appl. Anal. 2014, 2014, 594190.

25. Li, T.; Rogovchenko, Y.V. Oscillation of second-order neutral differential equations. Math. Nachr. 2015, 288, 1150-1162. [CrossRef]

26. Li, Q.; Wang, R.; Chen, F.; Li, T. Oscillation of second-order nonlinear delay differential equations with nonpositive neutral coefficients. Adv. Differ. Equ. 2015, 2015, 35. [CrossRef]

27. Li, T.; Rogovchenko, Y.V. Oscillation criteria for second-order superlinear Emden-Fowler neutral differential equations. Monatsh. Math. 2017, 184, 489-500. [CrossRef] 